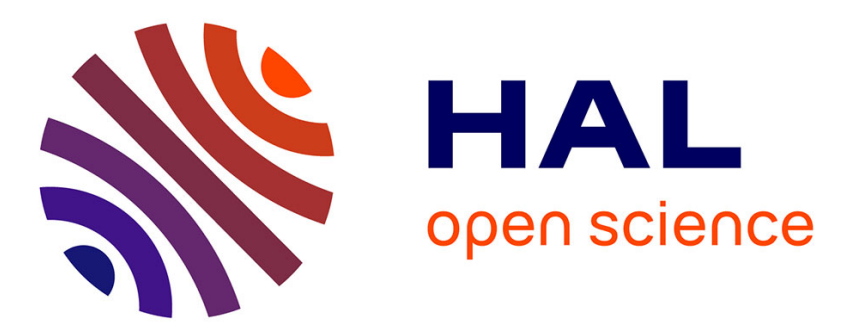

\title{
Convergence acceleration using the residual shape technique when solving structure-acoustic coupling with the Patch Transfer Functions method
}

\author{
M. Aucejo, Laurent Maxit, N Totaro, J.-L. Guyader
}

\section{- To cite this version:}

M. Aucejo, Laurent Maxit, N Totaro, J.-L. Guyader. Convergence acceleration using the residual shape technique when solving structure-acoustic coupling with the Patch Transfer Functions method. Computers \& Structures, 2010, 88 (11-12), pp.728-736. 10.1016/j.compstruc.2010.02.010 . hal-01668981

\section{HAL Id: hal-01668981 \\ https://hal.science/hal-01668981}

Submitted on 14 Mar 2019

HAL is a multi-disciplinary open access archive for the deposit and dissemination of scientific research documents, whether they are published or not. The documents may come from teaching and research institutions in France or abroad, or from public or private research centers.
L'archive ouverte pluridisciplinaire HAL, est destinée au dépôt et à la diffusion de documents scientifiques de niveau recherche, publiés ou non, émanant des établissements d'enseignement et de recherche français ou étrangers, des laboratoires publics ou privés. 


\title{
Convergence acceleration using the residual shape technique when solving structure-acoustic coupling with the Patch Transfer Functions method
}

\author{
M. Aucejo ${ }^{1}$, L. Maxit, N. Totaro, J.-L. Guyader \\ Laboratoire Vibrations Acoustique, INSA Lyon, 25 bis avenue Jean Capelle 69621 \\ Villeurbanne Cedex, FRANCE
}

\begin{abstract}
The forced response of the structure-water-filled cavity system is investigated from the Patch Transfer Functions method. In such a case, a poor convergence of the PTF method is observed when using standard mode expansion to build the cavity-PTF. To improve its convergence and maintain the advantages of substructuring, residual shapes are introduced in the cavity-PTF computation, which is the new material of this article. This technique is successfully applied on numerical examples, highlighting the interest of such an approach, especially in heavy fluid.
\end{abstract}

Key words: Strong coupling, PTF, FEM, residual modes

\section{Introdution}

The forced response of a structure backed by an acoustic cavity has been studied extensively during the last few decades. Classical models are based on the coupling of in vacuo structural modes and rigid-walled cavity modes, providing comprehensive formulations to solve the coupled system $[1,2,3]$. These models introduce a modal cross-coupling term to describe the interaction between the structure and the cavity. Sum and Pan [4] have analyzed this interaction term to underline the physical mechanisms of cross-coupling.

When considering a geometrically complex system, analytical methods are often replaced by the Finite Element Method (FEM). However, the direct resolution of the coupled problem is time consuming. To overcome this difficulty, model reduction techniques were developed such as the Component Mode Synthesis (CMS) methods $[5,6]$. These methods, based on the modal approach, allow describing the dynamics of the coupled system with a reduced but sufficient

\footnotetext{
*Corresponding author. Fax : 33.4.72.43.87.12. E-mail address: mathieu.aucejo@insalyon.fr
} 
number of degrees of freedom. In the case of strong coupling, the use of uncoupled modal bases can lead to poor convergence if the coupling between the high order modes of a subsystem with low order modes of the other system is not considered in the computation. To improve the convergence of the modal approach, several techniques were developed, such as pseudostatic correction $[7,8,9]$ and residual modes $[10,11,12]$. The aim of these techniques is to obtain simple and efficient reduction bases to maintain the advantages of modal superposition while improving its accuracy.

In the present paper, the Patch Transfer Functions (PTF) method is used to solve the structure-cavity coupling, when the acoustic cavity is filled with water. This method developed by Maxit et al. [13] is a tool for predicting the vibro-acoustic response of complex systems coupled through surfaces divided into elementary areas called patches. This approach, based on substructuring $[14,6]$ and the impedance and mobility approach [15], consists in studying each subsystem independently in order to build a set of transfer functions defined by using mean values on the patches, called Patch Transfer Functions. Then, assembling PTF by using the continuity relations leads to the fast resolution of the coupled problem. However, as presented in this paper, the use of a standard mode expansion to build the cavity-PTF leads to poor convergence of the PTF method in heavy fluid.

To alleviate this problem, we introduce the residual mode technique in the present approach. This novelty allows improving the convergence of the coupled system response and maintain the advantages of substructuring, by computing a residual shape basis for the rigid-walled cavity independently of the structure. Classically, these residual shapes, which are attachment modes, correspond to the response of the cavity subjected to structural mode excitation. Nevertheless, in the PTF method, the residual shapes correspond to the response of the cavity excited by a constant normal displacement imposed on a patch at a specific frequency. This ensures the convergence of the proposed method.

In the following sections, the theoretical background of the proposed method is first recalled and the PTF computation is then derived from the FE formulation of each subsystem is developed. In sections 5 and 6 , the structural-acoustic problem is considered by comparing the forced response of a plate coupled to a water-filled or air-filled cavity, either obtained by a direct resolution using FEM, or by the PTF method including the residual mode technique. The introduction of the residual shapes concept in cavity-PTF computation allows obtaining good results with a coarser interface mesh than that used for standard FEM. Finally, to illustrate the advantage of the present approach on an industrial case, the radiation of a bulkhead two ballasts is considered. The influence of residual shapes is studied by comparing PTF results obtained either from a direct computation of the PTF of both ballasts or a mode expansion with or without the introduction of residual shapes in the calculations. 


\section{Description of the vibro-acoustic problem}

Let us consider a structure backed by an acoustic cavity with rigid walls and excited by a point force $F$ on its surface $S_{v}$ as presented in Fig. (1). The surface $S_{v}$ can also be identified as the coupling surface of the structure-cavity system.

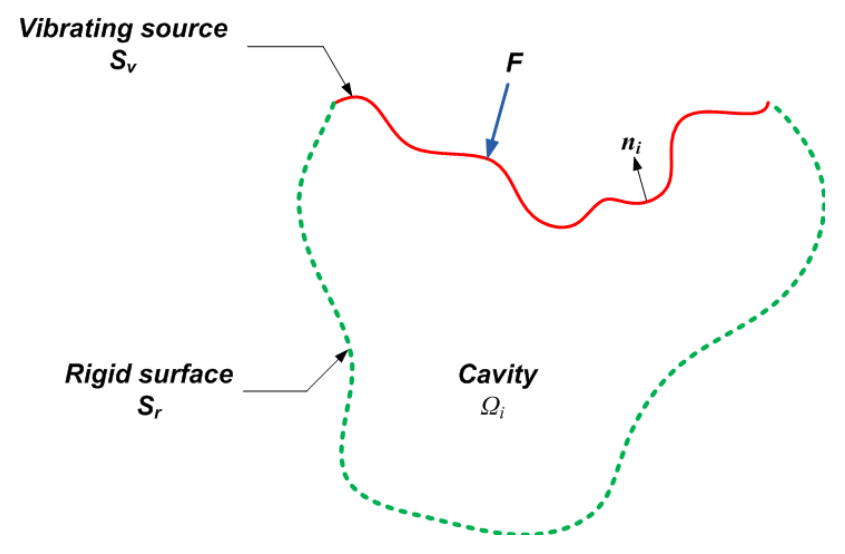

Figure 1: Basic vibro-acoustic problem

The coupled structural problem is governed by its equation of motion with boundary conditions imposed on the structure boundary $\partial S_{v}$. The structure is excited by a harmonic point force $\{F\}$ and the cavity pressure-induced force $\left\{F_{c}\right\}=-[A]\{P\}$ acting on the structure. The displacement of the structure is harmonic $\left\{U_{s}(M, t)\right\}=\left\{U_{s}(M)\right\} e^{j \omega t}$ and satisfies the following equation:

$$
\left[K_{s}-\omega^{2} M_{s}\right]\left\{U_{s}\right\}=\{F\}-[A]\{P\}
$$

where $M_{s}$ and $K_{s}$ are the mass and stiffness matrices of the structure, $A$ the coupling matrix of the structure-cavity system and $\omega$ the radian frequency.

The coupled acoustic cavity problem is governed by the homogeneous Helmholtz equation. Rigid-walled boundary conditions are imposed on the rigid surface $S_{r}$ and a source term $Q_{s}=-\omega^{2}[A]^{T}\left\{U_{s}\right\}$ resulting from the vibration of the structure is imposed on $S_{v}$. The acoustic pressure in the cavity is harmonic $\{P(M, t)\}=\{P(M)\} e^{j \omega t}$ and satisfies the following equation:

$$
\left[K_{f}-\omega^{2} M_{f}\right]\{P\}=-\omega^{2}[A]^{T}\left\{U_{s}\right\}
$$

where $M_{f}$ and $K_{f}$ are the mass and stiffness matrices of the acoustic cavity.

Eqs. (1) and (2) leads to the linear matrix system given by the following equation:

$$
\left(\left[\begin{array}{cc}
K_{s} & A \\
0 & K_{f}
\end{array}\right]-\omega^{2}\left[\begin{array}{cc}
M_{s} & 0 \\
-A^{T} & M_{f}
\end{array}\right]\right)\left\{\begin{array}{c}
U_{s} \\
P
\end{array}\right\}=\left\{\begin{array}{c}
F \\
0
\end{array}\right\}
$$


Eq. (3) shows that for the general structure-acoustic problem, the system to be solved has a non-symmetric mass and stiffness matrices. This lack of symmetry leads to difficulties in computing the dynamic response of the system, since Eq. (3) is costly to solve. Likewise, the extraction of eigenvalues for large structure-acoustic systems can be difficult with this non-symmetric formulation. To alleviate these difficulties, several symmetrization procedures have been developed. These procedures are based on different approaches, namely the displacement and pressure $(u, p)$ formulation or displacement, pressure and vorticity moment $(u, p, \Lambda)$ formulation $[16,17]$, the velocity potential formulation $[18,19]$ and the displacement formulation [20]. Finally, it should be noted that the matrix system given by Eq. (3) is not used in the proposed method, since each subsystem is considered independently and coupled by continuity equations.

\section{Patch Transfer Functions method}

\subsection{Description of the method}

The PTF method [13, 21, 22] is a substructuring approach that allows computing the vibro-acoustic response of complex systems coupled through their common surfaces divided into elementary areas called patches. Then, the Patch Transfer Functions (PTF), corresponding to an impedance or a mobility are computed for each patch of the coupling surface and assembled by using the superposition principle and writing the continuity relations.

This technique is efficient and well adapted to complex systems, since each subsystem is defined by its own FE model and solved independently of the others. Consequently, modification of one of the subsystems only leads to a simple update of this subsystem, provided the geometry of the structure-cavity interface remains unchanged. Furthermore, the PTF method leads to small, symmetric systems fully populated with complex coefficients. Moreover, the impedance and mobility matrices, which are the basic quantities of the method, can be obtained by measurements, analytical modelling and numerical modelling (FEM, BEM, etc.). Consequently, parallel computation of the basic quantities for each subsystem is possible, since the basic quantities are established on uncoupled subsystems. This is one of the method's advantages, since different approaches can be combined to obtain the response of a multi-coupled complex system. Moreover, if the PTFs are derived from the FE formulation, the use of incompatible meshes at the interface of the subsystems is possible, since the problem of compatibility is solved by the patch mesh as illustrated by Fig. (2). The management is indeed permitted because the PTF method does not relate nodes values but values averaged over the surface of a patch. However, as presented in Fig. (2), the mesh of the subsystems can not be highly incompatible at the interface insofar as nodes have to be included in each patch.

Finally, the PTF method differs from standard substructuring methods, e.g. the Craig-Bampton fixed interface approach [23], since the subsystems are coupled through patches instead of nodes. It should also be noted that Ouisse et 


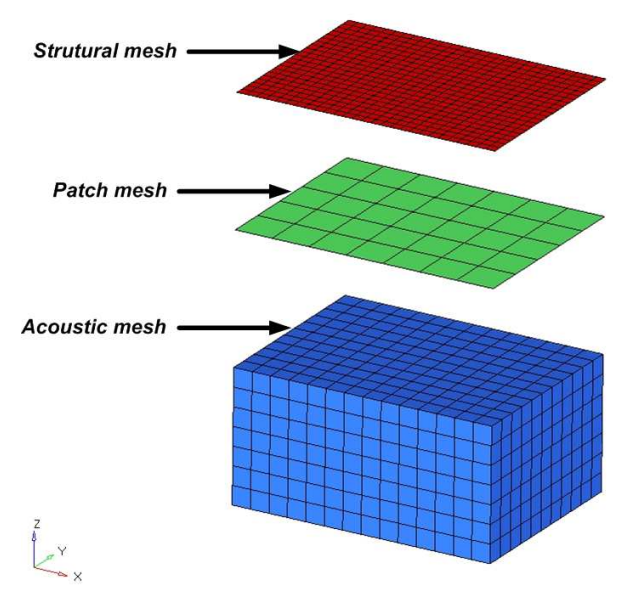

(a)

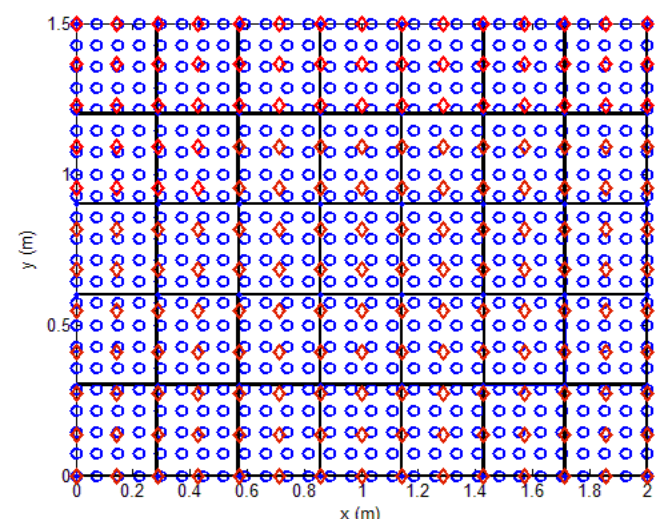

(b)

Figure 2: Use of incompatible mesh - (a) Definition of the PTF model and (b) Detection of FE nodes included in patches: ( $\circ$ ) nodes of the first subsystem and $(\diamond)$ nodes of the second subsystem 
al. [21] and Chazot and Guyader [24] have shown that the number of patches is lower than the number of interface nodes. As a consequence, the size of the PTF model is smaller than that obtained with the Craig-Bampton method. Indeed, the size of the reduced model is $N_{\text {patch }} \times N_{\text {patch }}$ ( $N_{\text {patch }}$ : Number of patches) for the PTF method, while for a Craig-Bampton method the size of the reduced model is $\left(N_{i m}+N_{i d o f}\right) \times\left(N_{i m}+N_{i d o f}\right)\left(N_{i m}\right.$ : Number of fixed interface modes chosen for each subsystem, $N_{\text {idof }}$ : Number of interface dofs).

\subsection{PTF definition}

In the case of the structure-cavity problem, the coupled system is divided into two subsystems, namely a structure and an acoustic cavity, and PTF are defined for each subsystem on the coupling surface $S_{v}$ divided into patches.

For the structure, the PTF $Y_{j k}^{s}$ between an excited patch $k$ and a receiving patch $j$ is the mobility defined as the ratio of the mean structural velocity $\bar{v}_{j}^{s}$ on patch $j$ and the mean pressure $\bar{p}_{k}^{s}$ on patch $k$ (see Eq. (4)),

$$
Y_{j k}^{s}=\frac{\bar{v}_{j}^{s}}{\bar{p}_{k}^{s}}
$$

where $\bar{\bullet}_{p}=\frac{1}{S_{p}} \int_{S_{p}} \bullet d S_{p}$ is the space-averaged quantity on the patch $p$.

For the acoustic cavity, the PTF $Z_{j k}^{c}$ between an excited patch $k$ and a receiving patch $j$ is the impedance defined as the ratio of the mean acoustic pressure $\bar{p}_{j}^{c}$ on patch $j$ and the mean normal velocity $\bar{v}_{k}^{c}$ on patch $k$ (see Eq. $(5))$,

$$
Z_{j k}^{c}=\frac{\bar{p}_{j}^{c}}{\bar{v}_{k}^{c}}
$$

\subsection{Subsystems coupling}

Coupling the subsystems is performed by writing continuity conditions on each patch of the coupling surface $S_{v}$ as expressed in Eq. (6) for a patch $j$.

$$
\left\{\begin{array}{l}
\bar{p}_{j}^{s}=\bar{p}_{j}^{c}=\bar{p}_{j} \\
\bar{v}_{j}^{s}=\bar{v}_{j}^{c}=\bar{v}_{j}
\end{array}\right.
$$

These coupling conditions are then used to determine the coupling normal velocity $\bar{v}_{j}$ on each patch $j$. For this purpose, the linearity of the problem is used. The normal structural velocity $\bar{v}_{j}^{s}$ on the patch $j$ is thus defined as the superposition of the in vacuo structural velocity $\widetilde{\bar{v}}_{j}^{s}$ (uncoupled structural response) and the normal velocity $Y_{j k}^{s} \bar{p}_{k}$ due to the pressure caused by the acoustic cavity on the structure (see Eq. (7)),

$$
\bar{v}_{j}^{s}=\widetilde{\bar{v}}_{j}^{s}+Y_{j k}^{s} \bar{p}_{k}
$$


Similarly, the pressure $\bar{p}_{j}^{c}$ on patch $j$ of the cavity can be calculated by using linearity, as the superposition of the pressure created by the acoustic source inside the cavity $\widetilde{\bar{p}}_{j}^{c}$ and the pressure due to structural vibrations $Z_{j k}^{c} \bar{v}_{k}$ (see Eq. $(8))$.

$$
\bar{p}_{j}^{c}=\widetilde{p}_{j}^{c}+Z_{j k}^{c} \bar{v}_{k}
$$

By introducing the coupling conditions (Eq. (6)), simple mathematical operations make it possible to obtain the coupling normal velocity $\bar{v}_{j}$ as presented in Eq. (9) in the absence of acoustic sources in the cavity.

$$
\bar{v}_{j}=\left(\mathbb{I}-Y_{j i}^{s} Z_{i k}\right)^{-1} \widetilde{v}_{k}^{s}
$$

where $\mathbb{I}$ is the identity matrix.

\section{PTF computation from a FE formulation and standard mode ex- pansion}

\subsection{Structure-PTF computation}

Defining the structure-PTF requires determining the structural velocity when a constant pressure $\bar{p}_{k}$ is imposed on patch surface $S_{k}$. Consequently, Eq. (10) has to be solved for each patch $k$ of the vibrating structure.

$$
\left[K_{s}-\omega^{2} M_{s}\right]\left\{U_{s}\right\}=\left\{F_{k}\right\}
$$

In Eq. (10), the excitation term $F_{k}$ corresponding to the constant pressure $\bar{p}_{k}$ imposed on the patch $k$ is given by the following equation:

$$
F_{k}= \begin{cases}\frac{A_{k}}{N} \bar{p}_{k} & \text { on } S_{k} \\ 0 & \text { on } S_{v} \backslash S_{k}\end{cases}
$$

where $A_{k}$ is the area of patch $k$ and $N$ is the number of FE nodes included in patch $k$.

Eq. (11) is solved by standard mode expansion. For this purpose, displacement $\left\{U_{s}\right\}$ is expanded on its structural modes as expressed in the following equation:

$$
\left\{U_{s}\right\}=\left[\psi_{n}\right]\left\{u_{n}\right\}
$$

where $\left[\psi_{n}\right]$ is the mode shape matrix and $\left\{u_{n}\right\}$ is the modal amplitude vector.

The generalized mass $\left[M_{s n}\right]$, stiffness $\left[K_{s n}\right]$ and force $\left[F_{n}\right]$ are defined as presented in the following equation:

$$
\left\{\begin{array}{l}
{\left[K_{s n}\right]=\left[\psi_{n}\right]^{T}\left[K_{s}\right]\left[\psi_{n}\right]} \\
{\left[M_{s n}\right]=\left[\psi_{n}\right]^{T}\left[M_{s}\right]\left[\psi_{n}\right]} \\
\left\{F_{n}\right\}=\left[\psi_{n}\right]^{T}\left\{F_{f}\right\}
\end{array}\right.
$$


The reduced system presented in Eq. (14) is therefore solved at each frequency to obtain structural displacement $\left\{U_{s}\right\}$.

$$
\left[K_{s n}-\omega^{2} M_{s n}\right]\left\{u_{n}\right\}=\left\{F_{n}\right\}
$$

This procedure is repeated for each excited patch $k$ leading to the structural velocity matrix $[V]$ of dimensions $N_{d o f} \times N_{\text {patch }}$, where $N_{d o f}$ is the number of degrees of freedom of the structure and $N_{\text {patch }}$ the number of patches.

The structure-PTF $Y_{j k}^{s}$ is thus obtained by averaging the normal velocities for the nodes included in the receiving patch $j$.

\subsection{Cavity-PTF computation}

The pressure response to a unit normal velocity $\bar{v}_{p}^{c}$ imposed on the patch surface $S_{p}$ has to be computed for each patch $p$ of the coupling surface of the acoustic cavity, leading to the solution of the following equation:

$$
\left[K_{f}-\omega^{2} M_{f}\right]\{P\}=\left\{Q_{p}\right\}
$$

In Eq. (15), the excitation term $Q_{p}$ corresponding to the constant normal velocity $\bar{v}_{p}^{c}$ imposed on the patch $p$ is given by the following equation:

$$
Q_{p}= \begin{cases}-j \omega \frac{A_{p}}{N} \bar{v}_{p}^{c} & \text { on } S_{p} \\ 0 & S_{v} \backslash S_{p}\end{cases}
$$

where $A_{p}$ is the area of patch $p$ and $N$ is the number of FE nodes included in patch $p$.

In this case too, Eq. (15) is solved by standard mode expansion, meaning that the acoustic pressure $\{P\}$ is expanded on its rigid-walled cavity modes, as shown in the following equation:

$$
\{P\}=\left[\phi_{n}\right]\left\{p_{n}\right\}
$$

where $\left[\phi_{n}\right]$ is the mode shapes matrix and $\left\{p_{n}\right\}$ is the modal amplitudes vector.

We now use the same resolution procedure as that used to compute the structure-PTF $Y_{j k}^{s}$ to obtain the cavity-PTF $Z_{j k}^{c}$.

\subsection{Concluding remarks}

The equations developed in this section are general, since they are based on FE models. Consequently, the approach can be applied to complex systems.

Furthermore, the standard mode expansion procedure has been used up now to compute the PTF without introducing the residual shapes concept. As demonstrated in the following sections, convergence of the method is difficult to achieve for acoustic cavities filled with heavy fluids such as water. In this case, residual shapes are computed (see section 6) to enrich the original cavity modal basis and improve the convergence of the method. 


\section{Structure-cavity coupling}

In this section, the PTF method is used to compute the forced response of a structure-cavity system and compared with standard FEM calculation.

To achieve this, we consider the academic case of a rectangular simply-supported plate excited by a point force $F$ and coupled to a parallelepiped rigid-walled cavity filled either with air, or water, as shown in Fig. (3). This example is voluntarily simplified in order to facilitate studying the convergence parameters of both methods, namely the interface FE mesh size and the number of modes taken into account in the cavity-PTF computation. The simulation parameters are listed in table 1.

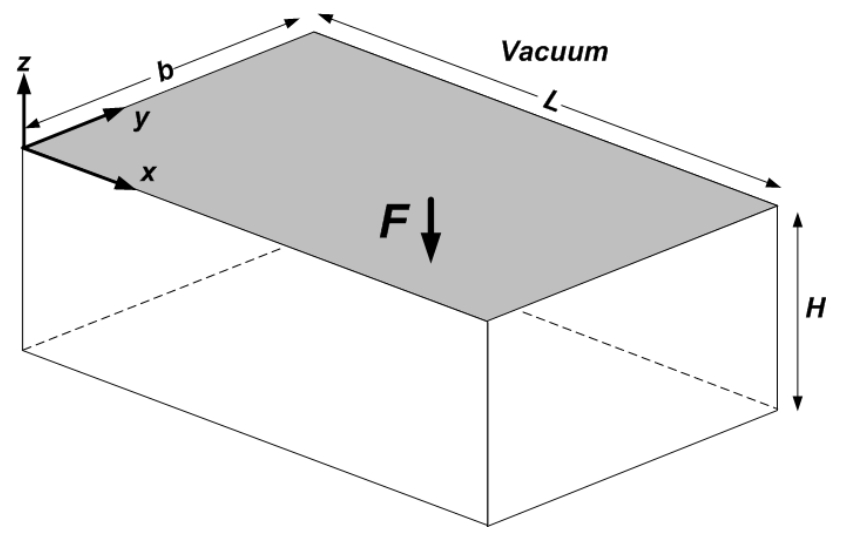

Figure 3: Definition of the rectangular simply-supported plate excited by a point force $F$ and coupled to a parallelepiped water-filled cavity

Table 1: Simulation parameters

\begin{tabular}{ll}
\hline Parameters & Values \\
\hline Plate length & $L=0.5 \mathrm{~m}$ \\
Plate width & $b=0.4 \mathrm{~m}$ \\
Plate thickness & $h=0.002 \mathrm{~m}$ \\
Cavity depth & $H=1 \mathrm{~m}$ \\
Young modulus & $E=2.1 \times 10^{11} \mathrm{~N} \cdot \mathrm{m}^{-2}$ \\
Poisson's ratio & $\nu=0.3$ \\
Plate density & $\rho=7800 \mathrm{~kg} \cdot \mathrm{m}^{-3}$ \\
Damping factor & $\eta=0.02$ \\
Air Density & $\rho_{0}=1.2 \mathrm{~kg} \cdot \mathrm{m}^{-3}$ \\
Sound speed in air & $c=340 \mathrm{~m} \cdot \mathrm{s}^{-1}$ \\
Water density & $\rho_{0}=1000 \mathrm{~kg} \cdot \mathrm{m}^{-3}$ \\
Sound speed in water & $c=1500 \mathrm{~m} \cdot \mathrm{s}^{-1}$ \\
\hline
\end{tabular}




\subsection{Convergence of FEM}

Before using the FE result as a reference, it is important to study the convergence of the method in order to define the optimal interface mesh criterion. To do this, a coincident mesh is used at the structure-cavity interface and a direct resolution of the coupled problem using NASTRAN is performed to avoid modal truncation effects on the results presented. The interface mesh criterion, which provides the size of the finite elements at the structure-cavity interface, is based on the smallest wavelength $\lambda$ of the uncoupled plate and cavity and computed at the maximal frequency of the range of interest $f_{\max }$ (see Eq. (18)).

$$
\left\{\begin{array}{l}
\lambda_{s}=\sqrt{2 \pi} f_{\max }^{-1 / 2} \sqrt[4]{\frac{E h^{2}}{12 \rho\left(1-\nu^{2}\right)}} \\
\lambda_{f}=\frac{c}{f_{\max }} \\
\lambda=\min \left(\lambda_{s}, \lambda_{f}\right)
\end{array}\right.
$$

Four interface mesh criteria are used to study the convergence of FEM $\left(\frac{\lambda}{6}\right.$, $\frac{\lambda}{12}, \frac{\lambda}{28}$ and $\frac{\lambda}{60}$ ) when the plate is coupled to a water-filled cavity and the mean square velocity of the plate is computed for each of them. This measurement is usually used in vibro-acoustics (see Ref. [6]) and provides an overview of the response of a system. Fig. (4) shows that convergence in heavy fluid requires a very fine interface mesh to capture the interface dynamics, since convergence occurs for a $\frac{\lambda}{28}$ mesh criterion. This difficulty is linked to coupling with heavy fluids such as water. For a cavity filled with air, convergence is much better and the usual $\frac{\lambda}{6}$ mesh criterion is adequate to properly describe the response of the coupled system.

The presence of the cavity leads to an added mass effect on the plate that decreases the flexural wave speed and thus the flexural wavelength. As a consequence, the standard $\frac{\lambda}{6}$ interface mesh criterion, based on the wavelength of the uncoupled subsystems, leads to an underestimation of the size of the interface finite elements necessary for describing the behavior of the coupled system.

\subsection{Convergence of PTF method}

\subsubsection{Light fluid case}

Ouisse et al. demonstrated in [21] that a $\frac{\lambda}{2}$ patch mesh criterion for the coupling surface was sufficient to obtain good results when two acoustic air-filled cavities are coupled. However, this patch mesh criterion is also valid for the structure-cavity coupling presented in Fig. (5). In this example, the simulation parameters are given in table 1. Furthermore, the structural modal basis contains 12 modes, corresponding to resonance modes below $508 \mathrm{~Hz}$, while the acoustic basis contains 9 modes, corresponding to resonance frequency modes below $515 \mathrm{~Hz}$.

In Fig. (5), the plate response is presented for a classical FE computation with a $\frac{\lambda}{6}$ mesh criterion and a PTF calculation with a patch size of $\frac{\lambda}{2}$ that ensures 


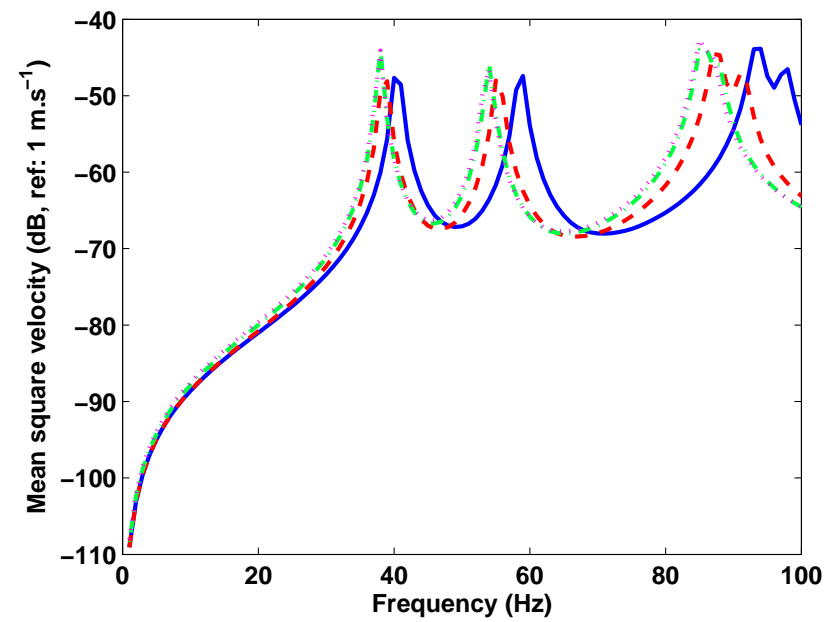

Figure 4: Convergence of FEM in heavy fluid - Comparison of the mean square velocity obtained by direct resolution for 4 interface mesh criteria: (-) $\frac{\lambda}{6},(--) \frac{\lambda}{12},(-.-) \frac{\lambda}{28}$ and (...) $\frac{\lambda}{60}$

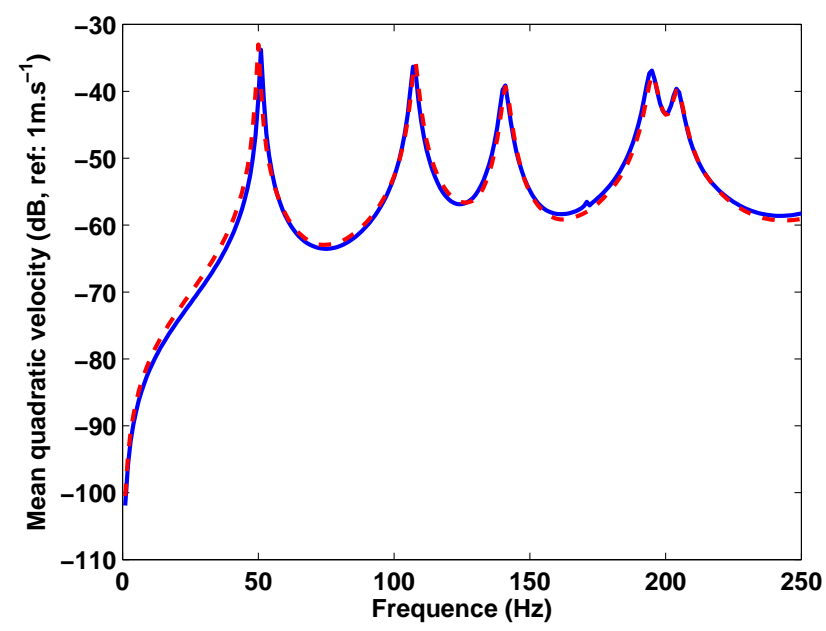

Figure 5: Convergence of PTF method in light fluid - Comparison of mean square velocity for: (-) FEM solution (interface element size: $\frac{\lambda}{6}$ ), (- -) PTF method (patch size: $\frac{\lambda}{2}$ )

the convergence of the proposed method. Consequently, the PTF method gives good results with a patch mesh coarser than a standard FE interface mesh. 


\subsubsection{Heavy fluid case}

Unlike the light fluid case, in heavy fluid, account has to be taken of the decrease of wavelength described in section 5.1, since the structure-cavity coupling is strengthened. Consequently, we used a $\frac{\lambda}{6}$ patch mesh criterion, i.e. 56 patches. To avoid a mode truncation effect, structure-PTF and cavity-PTF are obtained by direct resolution of Eqs. (10) and (15).

Fig. (6) shows that in this case too the PTF method gives satisfying results for a patch mesh coarser than the interface FE mesh.

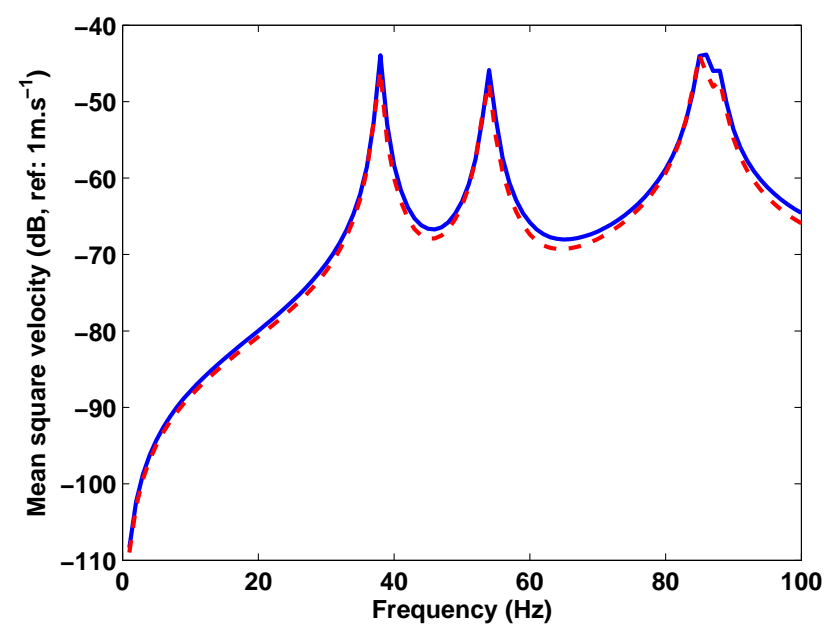

Figure 6: Convergence of the PTF method in heavy fluid - Comparison of mean square velocity for: (-) FEM solution (interface element size: $\frac{\lambda}{28}$ ), (- -) PTF method (patch size: $\frac{\lambda}{6}$ )

\section{Introduction of residual shapes in the cavity-PTF computation}

6.1. Influence of the mode truncation effect on the cavity-PTF computation in heavy fluid

As in section 5.2.2, the PTF can be obtained by direct resolution of FE models. However, when the size of the model is large, it is preferable to reduce it by using mode expansion.

Unfortunately, in heavy fluid the results obtained are sensitive to the truncation effect of acoustic cavity modes, as shown in Fig. (7). 200, 300 and 400 acoustic cavity modes, corresponding to resonant frequency modes below $8.8 \mathrm{kHz}, 10.5$ $\mathrm{kHz}$ and $11.7 \mathrm{kHz}$, were used to compute the cavity-PTF $Z_{j k}^{c}$, while 5 structural modes, corresponding to resonant frequency modes below $205 \mathrm{~Hz}$, were used to compute the structure-PTF $Y_{j k}^{s}$. This was sufficient to ensure its convergence since unchanged results were observed with more structural modes. This figure 
shows that more than 400 acoustic cavity modes are required to ensure the convergence of the PTF method.

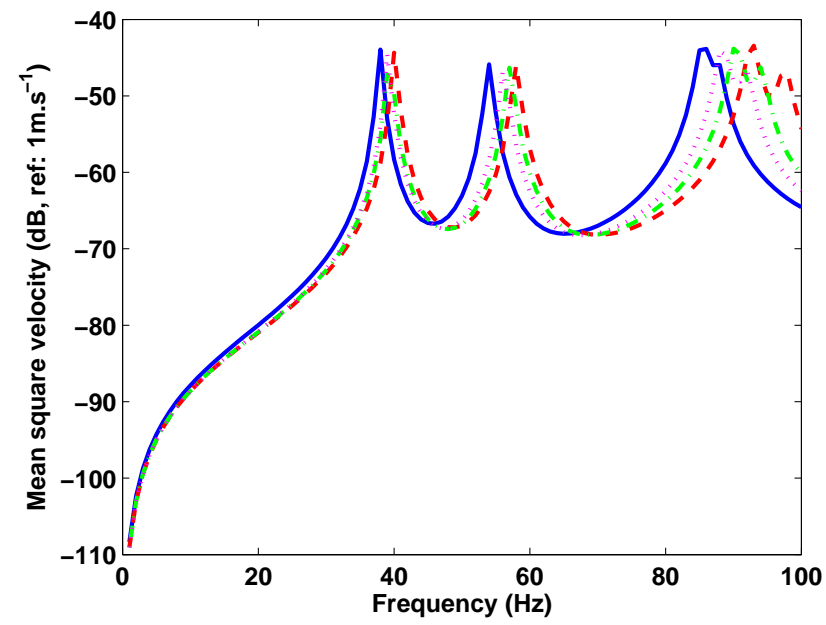

Figure 7: Truncation effect of acoustic cavity modes - Comparison of mean square velocity for: (-) FEM solution (interface element size: $\frac{\lambda}{28}$ ), (- -) PTF method for 200 acoustic cavity modes (patch size: $\frac{\lambda}{6}$ ), (-.-) PTF method for 300 acoustic cavity modes (patch size: $\frac{\lambda}{6}$ ) and (...) PTF method for 400 acoustic cavity modes (patch size: $\frac{\lambda}{6}$ )

\subsection{Introduction of residual shapes - methodology}

To achieve convergence of the PTF method in heavy fluid, high-order cavity modes are very important to provide a good description of the coupling mechanism. To overcome this difficulty, the concept of residual modes (see Ref. $[25,26])$ is used for the cavity-PTF computation. The residual shapes do not correspond to physical normal modes but allow enriching the modal basis with a set of functions describing the contribution of high-order modes.

In the PTF method, the idea consists in computing the residual shapes or attachment modes for the acoustic cavity independently of the structure in order to keep the substructuring aspect of the method. In the standard residual mode technique, the residual functions for an acoustic cavity correspond to the response of the cavity excited by structural normal modes [10, 11]. Consequently, a modification of the structure leads to a modification of structural normal modes that requires computing the residual shapes again. In our case, a residual shape corresponds to the response of the cavity excited by a constant normal displacement imposed on a patch $p$ at a specific radian frequency $\omega_{c}$. Consequently, a structural modification does not change the residual cavity shapes as long as the patch mesh remains unchanged. Furthermore, this definition leads to a number of residual shapes equal to the number of patches. This is a limitation of the method in the high frequency range, since the number of patches 
increases with frequency.

Using the notations used in section 4.2, the residual shapes $\left\{P_{r}\right\}$ of the cavity are computed by the direct resolution of the following equation:

$$
\left[K_{f}-\omega_{c}^{2} M_{f}\right]\left\{P_{r}\right\}=\left\{Q_{p}\right\}
$$

In Eq. (19), the excitation term $Q_{p}$ corresponding to the unit normal displacement $\bar{u}_{p}^{c}$ imposed on the patch $p$ is given by the following equation:

$$
Q_{p}= \begin{cases}\omega_{c}^{2} \frac{A_{p}}{N} \bar{u}_{p}^{c} & \text { on } S_{p} \\ 0 & \text { on } S_{v} \backslash S_{p}\end{cases}
$$

Thus we obtain a new reduction basis containing the $N$ physical modes kept in the original basis, enriched by the $N_{r}$ residual shapes $\left\{P_{r}\right\}$ (see Eq. (21)),

$$
[T]=\left[\phi_{1}, \ldots, \phi_{N} \mid P_{1}, \ldots, P_{N_{r}}\right]
$$

The reduction basis $[T]$ has to be reorthogonalized to maintain the validity of the mass and stiffness orthogonality relations. To do this, the reduced eigenvalue problem given by Eq. (22) has to be solved [27],

$$
\left([T]^{T}\left[K_{f}\right][T]-\omega^{2}[T]^{T}\left[M_{f}\right][T]\right)\left\{\varphi_{R}\right\}=\{0\}
$$

Finally, Eq. (22) leads to a new reduction basis $\{\Phi\}$ containing $N$ physical modes and $N_{r}$ residual shapes (see Eq. (23)).

$$
\{\Phi\}=[T]\left\{\varphi_{R}\right\}
$$

An example of the residual shape computed from Eq. (23) is plotted in Fig. (8). As expected, the residual shape varies slowly along the three directions of the cavity. It can also be seen that the residual pressure shape reaches its maximum in the vicinity of the excited patch whose center coordinates are $(\mathrm{x}, \mathrm{y}, \mathrm{z})=(0.22 \mathrm{~m}, 0.14 \mathrm{~m}, 0 \mathrm{~m})$.

\subsection{Numerical results}

In the example presented in this article, the new reduction basis contains 2 physical modes, corresponding to resonant frequency modes below $750 \mathrm{~Hz}$, and 56 residual shapes computed for $\omega_{c}=942.5 \mathrm{rad}^{-1}{ }^{-1}(150 \mathrm{~Hz})$. When using the residual shapes, the mean square velocity computed by the PTF method with a $\frac{\lambda}{6}$ patch mesh is in very good agreement with the reference result obtained by FEM, contrary to that computed by the PTF method without residual shapes, as shown in Fig. (9). This figure shows the advantage of using residual shapes, since it provides a visibly spectacular improvement of convergence.

Fig. (9) shows that the PTF method gives good results with a coarser interface mesh than that required in standard FEM to ensure the convergence of the method. The better convergence of the PTF method can be explained in part by the independent convergence of each subsystem obtained with a 


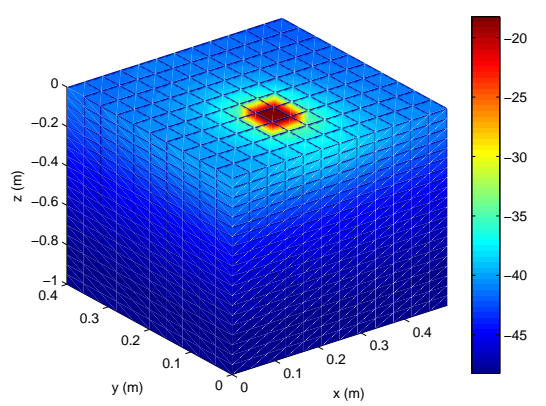

(a)

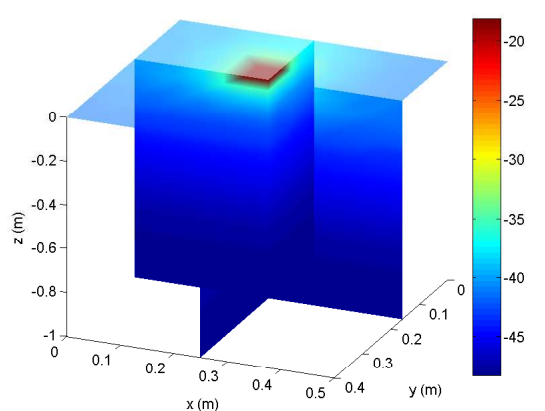

(b)

Figure 8: Example of residual pressure shape - Spatial distribution of acoustic pressure (a) on boundaries of the cavity and (b) in the cavity

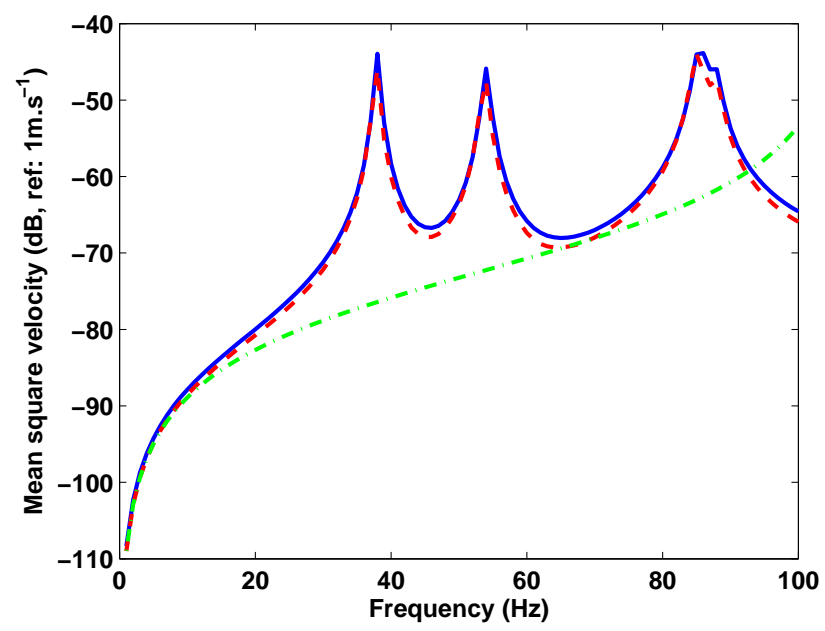

Figure 9: Residual shapes effect - Comparison of mean square velocity for: (-) FEM solution (interface element size: $\left.\frac{\lambda}{28}\right),(-.-)$ PTF method without residual shapes (patch size: $\frac{\lambda}{6}$ ) and (-) PTF method introducing residual shapes with $\omega_{c}=942.5 \mathrm{rad}_{\mathrm{s}} \mathrm{s}^{-1}$ (patch size: $\frac{\lambda}{6}$ )

standard $\frac{\lambda}{6}$ mesh criterion and the coupling technique that couples subsystems through patches (surfaces) instead of nodes. The PTF method associated with the residual shapes technique therefore provides effective time-saving computation compared to standard FEM.

In the example shown in Fig. (9), the value of $\omega_{c}$ corresponds to $20 \%$ of the first non-zero cavity mode.

In fact the value that must be used to compute the residual shapes is not clearly established, since for Tran et al. [10] and Cuppens et al. [12], it re- 
spectively corresponds to $50 \%$ and $75 \%$ of the maximal frequency of the range of interest. However, to thoroughly study the influence of this parameter, the problem is solved for three values of $\omega_{c}: 314.1 \mathrm{rad} . \mathrm{s}^{-1}(50 \mathrm{~Hz}), 942.5 \mathrm{rad} . \mathrm{s}^{-1}$ $(150 \mathrm{~Hz})$ and $6,283.2 \mathrm{rad} \cdot \mathrm{s}^{-1}(1000 \mathrm{~Hz})$. It is important to bear in mind that, in the present case, physical acoustic cavity modes are computed up to $750 \mathrm{~Hz}$. In other words, the first two values of $\omega_{c}$ are below the frequency of the last physical mode kept in the modal basis, while in the last case $\omega_{c}$ is above this frequency.

Fig. (10) shows that the convergence of the PTF method is ensured for $\omega_{c}=$ $314.1 \mathrm{rad} . \mathrm{s}^{-1}$ and $\omega_{c}=942.5 \mathrm{rad} . \mathrm{s}^{-1}$, which is not at all the case for $\omega_{c}=6283.2$ rad.s ${ }^{-1}$, since the contribution of modes between 750 and $1000 \mathrm{~Hz}$ is absent. Thus, the choice of $\omega_{c}$ is not arbitrary. However, convergence is achieved as long as the value of this parameter remains below the frequency of the last physical mode retained to compute the cavity-PTF.

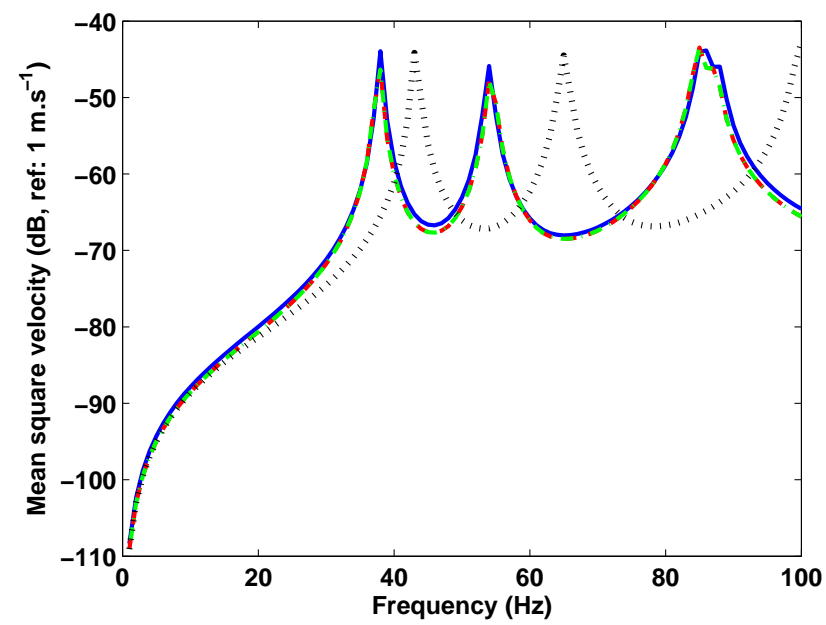

Figure 10: Influence of $\omega_{c}$ : (-) FEM solution, (- -) $\omega_{c}=314.1 \mathrm{rad} . \mathrm{s}^{-1}(50 \mathrm{~Hz}),(-.-) \omega_{c}=942.5$ rad.s ${ }^{-1}(150 \mathrm{~Hz})$ and $(..) \omega_{c}=6,283.2 \mathrm{rad} . \mathrm{s}^{-1}(1000 \mathrm{~Hz})$

\section{Radiation of a bulkhead into two ballasts}

The advantage of the residual shapes concept is illustrated on an industrial example. The proposed case concerns two water-filled ballasts, considered as rigid-walled acoustic volumes, which are separated by a clamped bulkhead mechanically excited, as illustrated in Fig. (11).

The first ballast has dimensions $4 \mathrm{~m}$ (width) by $2 \mathrm{~m}$ (height) by $2.75 \mathrm{~m}$ (depth) approximately, while the second ballast has dimensions $4 \mathrm{~m}$ (width) by $2 \mathrm{~m}$ (height) by $3.55 \mathrm{~m}$ (depth) approximately. Thus, the bulkhead, which is a steel shell of thickness $0.02 \mathrm{~m}$, has dimensions $4 \mathrm{~m} \times 2 \mathrm{~m}$ approximately. The excitation consists of a prescribed point force acting on the bulkhead along the x-axis. 
A FE model of the whole system is used to compute the basic quantities of the PTF method. Table 2 sums up the main features of the FE mesh of the whole system.

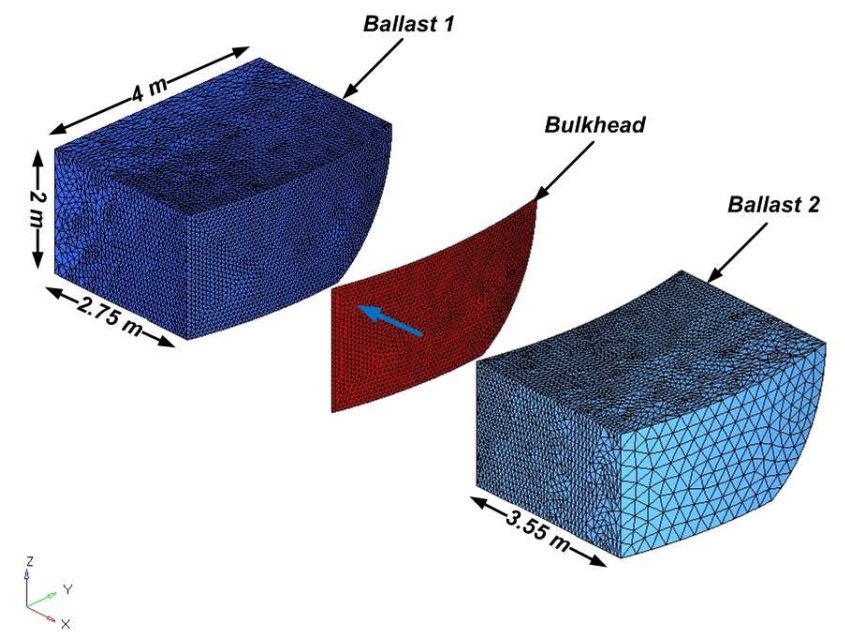

Figure 11: Definition of the FE model

Table 2: Main features of the FE mesh of the whole system

\begin{tabular}{cc}
\hline System & Number of degrees of freedom \\
\hline Ballast 1 & 27864 dofs \\
Ballast 2 & 29147 dofs \\
Shell & 9630 dofs \\
\hline
\end{tabular}

The aim is to build a PTF model in which both ballasts and the bulkhead are modeled by their PTF. Then, the influence of residual shapes for both ballasts is studied by comparing the pressure level at one point of each ballast, when the cavity-PTF are obtained either from a direct computation using FEM or a mode expansion with or without residual shapes. The structure-PTF are computed from a mode expansion insofar as they converge rapidly.

In the present case, the PTF equations, namely Eqs. (6)-(9), has to be rewritten to take into account the presence of the second ballast, corresponding to a third subsystem. To this end, continuity equations are rewritten as expressed by the following equation: 


$$
\left\{\begin{array}{l}
\bar{p}_{j}^{s}=\bar{p}_{j}^{b 1}-\bar{p}_{j}^{b 2} \\
\bar{v}_{j}^{s}=\bar{v}_{j}^{b 1}=-\bar{v}_{j}^{b 2}=\bar{v}_{j}
\end{array}\right.
$$

These continuity conditions are used to calculate the coupling normal velocity $\bar{v}_{j}$ on each patch $j$. Again, the introduction of continuity conditions in Eq. (7) allows obtaining the coupling normal velocity $\bar{v}_{j}$ given by the following equation:

$$
\bar{v}_{j}=\left[\mathbb{I}-Y_{j i}^{s}\left(Z_{i k}^{b 1}+Z_{i k}^{b 2}\right)\right]^{-1} \widetilde{\bar{v}}_{k}^{s}
$$

where $Z_{i k}^{b 1}$ is the cavity-PTF of the first ballast and $Z_{i k}^{b 2}$ is the cavity-PTF of the second ballast.

The pressure at point $L$ (resp. $M$ ) belonging to the first ballast (resp. the second one) is thus obtained from the coupling velocity by using the cavity-PTF $Z_{L j}$ calculated between the point $L$ and the patch $j$.

$$
\left\{\begin{array}{l}
p^{b 1}(L)=Z_{L j}^{b 1} \bar{v}_{j} \\
p^{b 2}(M)=Z_{L j}^{b 2} \bar{v}_{j}
\end{array}\right.
$$

PTF calculations have been performed for 329 patches. The results obtained from a direct computation of the cavity-PTF are compared in Fig. (12) with results yielded by a mode expansion of the cavity-PTF with or without introducing the residual shapes. It can be also noticed that the mode expansion have been performed using normal modes computed up to $1600 \mathrm{~Hz}$. This corresponds to 168 normal modes for the first ballast and 171 normal modes for the second one. Fig. (12) shows a very good agreement between a direct computation of the cavity-PTF and a modal expansion introducing the residual shapes, while the calculation using resonant modes only does not converge.

The introduction of residual shapes allows decreasing in computational efforts as highlighted in Table 3, where a comparison of total CPU times is performed for a direct computation of the cavity-PTF and a modal calculation with or without the residual shapes. This table shows that the total CPU time for a modal approach introducing the residual shapes is much lower than that of a direct calculation, while the accuracy is equivalent for both approach. It can be noticed that the CPU time for a modal calculation with or without the residual shapes are similar. Consequently, the introduction of residual shapes improves the accuracy of the modal approach, without significantly increasing the computational effort.

Table 3: Comparison of the total CPU times for the calculation of the cavity-PTF

\begin{tabular}{cccc}
\hline & direct & normal modes & normal modes + residual shapes \\
\hline Ballast 1 & $35223 \mathrm{~s}$ & $1423 \mathrm{~s}$ & $1456 \mathrm{~s}$ \\
Ballast 2 & $38103 \mathrm{~s}$ & $1456 \mathrm{~s}$ & $1465 \mathrm{~s}$
\end{tabular}




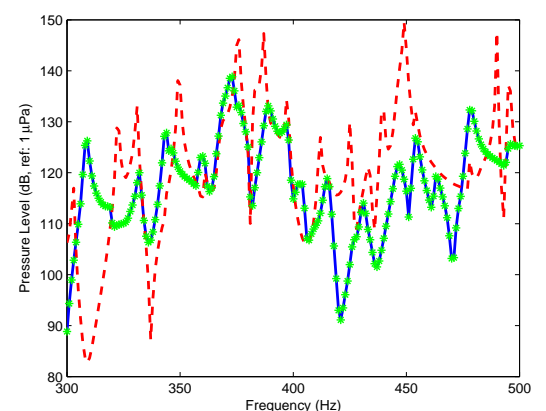

(a)

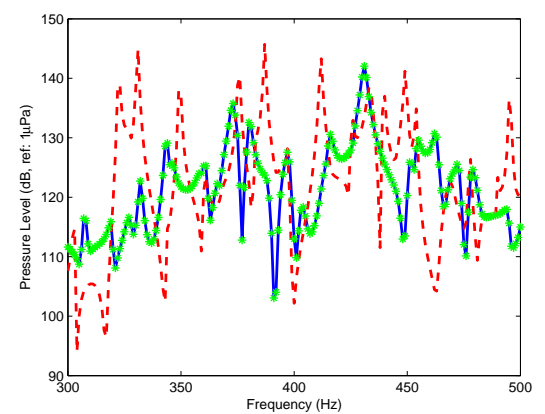

(b)

Figure 12: Influence of residual shapes - Comparison of the pressure level (a) at the point $(-2.55 \mathrm{~m}, 1.77 \mathrm{~m},-0.66 \mathrm{~m})$ of the first ballast and $(\mathrm{b})$ at the point $(0.46 \mathrm{~m}, 0.59 \mathrm{~m},-0.82 \mathrm{~m})$ of the second ballast when the cavity-PTF are obtained from (-) a direct calculation, (- -) a mode expansion without the residual shapes and $(*)$ a mode expansion with residual shapes

\section{Conclusion}

The PTF substructuring approach allows computing the response of structurecavity systems. It has the advantage of solving vibro-acoustic problems with reduced computational cost compared to standard FEM. The coupling technique consists in discretizing the coupling surface in elementary areas called patches on which continuity equations are verified. The novelty of the present paper concerns the introduction of the residual shapes concept in order to accelerate the convergence of the PTF method, when solving a structure-acoustic coupling in heavy fluid. The studies presented in this article show that the concept of residual modes associated with interface coupling patches permits substantially improving the convergence of the PTF approach while keeping the uncoupled resolution of equations for each subsystem.

\section{Acknowledgment}

The authors are grateful to the French Ministry of Defense for funding this work.

\section{References}

[1] EH Dowell, GF Gorman III, and Smith DA. Acoustoelasticity: General Theory, Acoustic Modes and Forced Response to Sinusoidal Excitation, including Comparisons with Experiment. Journal of Sound and Vibration, 52:519-542, 1977. 
[2] RW Guy and MC Bhattacharya. The Transmission of Sound Through a Cavity-Backed Finite Plate. Journal of Sound and Vibration, 27 (2):207216, 1973.

[3] L Gagliardini, J Roland, and J-L Guyader. The Use of a Functional Basis to Calculate Acoustic Transmission Between Rooms. Journal of Sound and Vibration, 145 (3):457-478, 1991.

[4] KS Sum and J Pan. On Acoustic and Structural Modal Cross-Couplings in Plate-Cavity Systems. Journal of the Acoustical Society of America, 107 (4):2021-2038, 2000.

[5] WC Hurty. Dynamic Analysis of Structural Systems using Component Modes. AIAA J., 3 (4):678-685, 1965.

[6] M Tournour, N Atalla, O Chiello, and Sgard F. Validation, Performance, Convergence and Application of Free Interface Component Mode Synthesis. Computers \& Structures, 79:1861-1876, 2001.

[7] HJP Morand and R Ohayon. Fluid-Structure Interaction. Wiley, New-York, 1995.

[8] R Ohayon. Reduced Models for Fluid-Structure Interaction Problems. Int. J. Numer. Meth. Engng, 60:139-152, 2004.

[9] M Tournour and N Atalla. Pseudostatic Corrections for the Forced Vibroacoustic Response of a Structure-Cavity System. Journal of the Acoustical Society of America, 107 (5):2379-2386, 2000.

[10] QH Tran, M Ouisse, and N Bouhaddi. Comparison of Robust CMS Methods for Damped Vibroacoustic Problems. In Proceedins of ISMA 2008, Leuven, Belgium, 2008.

[11] N Roy and M Lapi. Efficient Computation of the Radiated Sound Power of Vibrating Structures using a Modal Approach. In Proceedings of Acoustics'08, Paris, France, 2008.

[12] K Cuppens, P Sas, and L Hermans. Evaluation of the FRF Based Substructuring and Modal Synthesis Technique Applied to Vehicle FE Data. In Proceedings of ISMA 25, Leuven, Belgium, 2000.

[13] L Maxit, C Cacciolati, and J-L Guyader. Airborne Noise Prediction using Patch Acoustic Impedance. In Proceedings of ICSV 9, Orlando, UnitedStates, 2002.

[14] G Kergoulay, E Balmès, and D Clouteau. Model Reduction for Efficient FEM/BEM Coupling. In Proceedings of ISMA 25, Leuven, Belgium, 2000.

[15] SM Kim and MJ Brennan. A Compact Matrix Formulation using the Impedance and Mobility Approach for the Analysis of Structural-Acoustic Systems. Journal of Sound and Vibration, 223 (1):97-113, 1999. 
[16] KJ Bathe, C Nitikipaiboon, and X Wang. A Mixed Displacement-Based Finite Element Formulation for Acoustic Fluid-Structure Interaction. Computer \&3 Structures, 56, No. 2/3:225-237, 1995.

[17] X Wang and KJ Bathe. Displacement/Pressure Based Mixed Finite Element Formulations for Acoustic Fluid-Structure Interaction Problems. Int. J. Numer. Meth. Engng, 40:2001-2017, 1997.

[18] GC Everstine. A Symmetric Potential Formulation for Fluid-Structure Interactions. Journal of Sound and Vibration, 107 (1):121-129, 1981.

[19] LG Olson and KJ Bathe. Analysis of Fluid-Structure Interactions - A Direct Symmetric Coupled Formulation Based on a Fluid Velocity Potential. Computer \& Structures, 21:21-32, 1985.

[20] MA Hamdi, Y Ousset, and G Verchery. A Displacement Method for the Analysis of Vibrations of Coupled Fluid-Structure Systems. Int. J. Numer. Meth. Engng, 13:139-150, 1978.

[21] M Ouisse, L Maxit, C Cacciolati, and Guyader J-L. Patch Transfer Functions as a Tool to Couple Linear Acoustic Problems. Journal Vibration Acoustics, ASME transaction, 127:458-466, 2005.

[22] N Totaro, B Andro, C Peteul, and J-L Guyader. Extension of the Patch Transfer Functions Method (PTF Method) to High Frequency Domain (Sub-Cavities Decomposition). In Proceedings of Internoise 2007, Istanbul, Turkey, 2007.

[23] RR Craig and MCC Bampton. Coupling of Substructures for Dynamic Analysis. AIAA Journal, 6:1313-1319, 1968.

[24] J-D Chazot and J-L Guyader. Prediction of Transmission Loss of Double Panels with a Patch-Mobility Method. Journal of the Acoustical Society of America, 121 (1):267-278, 2007.

[25] RH MacNeal. A Hybrid Method of Component Mode Synthesis. Computers ES Structures, 1:581-601, 1971.

[26] S Rubin. Improved Component-Mode Representation for Structural Dynamic Analysis. AIAA Journal, 13:995-1006, 1975.

[27] E Balmès. Theoretical Course Notes: Methods for Vibration Design and Validation. In Ecole Centrale Paris, 1997-2008. 